\title{
Persistent chemosensory dysfunction in a young patient with mild COVID-19 with partial recovery 15 months after the onset
}

\author{
Maria Paola Cecchini ${ }^{1}$ (1) - Lorenzo Brozzetti ${ }^{2}$. Nicolò Cardobi ${ }^{3} \cdot$ Luca Sacchetto $^{4}$ - Davide Gibellini ${ }^{5}$. \\ Stefania Montemezzi ${ }^{3} \cdot$ Marta Cheli $^{6} \cdot$ Paolo Manganotti $^{6} \cdot$ Salvatore Monaco $^{2} \cdot$ Gianluigi Zanusso $^{2}$
}

Received: 22 May 2021 / Accepted: 24 September 2021 / Published online: 2 October 2021

(c) Fondazione Società Italiana di Neurologia 2021

\begin{abstract}
Objective It is reported that recovery from COVID-19 chemosensory deficit generally occurs in a few weeks, although olfactory dysfunction may persist longer. Here, we provide a detailed follow-up clinical investigation in a very young female patient (17-year-old) with a long-lasting anosmia after a mild infection, with partial recovery 15 months after the onset.

Methods Neuroimaging and neurophysiologic assessments as well as olfactory mucosa swabbing for microbiological and immunocytochemical analyses were performed. Olfactory and gustatory evaluations were conducted through validated tests. Results Chemosensory evaluations were consistent with anosmia associated with parosmia phenomena and gustatory impairment, the latter less persistent. Brain MRI $(3.0 \mathrm{~T})$ showed no microvascular injury in olfactory bulbs and brain albeit we cannot rule out slight structural abnormalities during the acute phase, and a high-density EEG was negative. Immunocytochemistry of olfactory mucosa swabs showed high expression of ACE2 in sustentacular cells and lower dot-like cytoplasmic positivity in neuronal-shaped cells.

Discussion The occurrence of long-term persistent olfactory deficit in spite of the absence of structural brain and olfactory bulb involvement supports the view of a possible persistent dysfunction of both sustentacular cells and olfactory neurons. The gustatory dysfunction even if less persisting for the described features could be related to a primary gustatory system involvement. Future longitudinal studies are needed to investigate the persistence of chemosensory impairment, which could have a relevant impact on the daily life.
\end{abstract}

Keywords COVID-19 $\cdot$ Persistent chemosensory deficit $\cdot$ Anosmia $\cdot$ Parosmia $\cdot$ Dysgeusia

Maria Paola Cecchini

mariapaola.cecchini@univr.it

1 Department of Neurosciences, Biomedicine, and Movement Sciences, Anatomy and Histology Section, University of Verona, Strada le Grazie, 837134 Verona, Italy

2 Department of Neurosciences, Biomedicine, and Movement Sciences, Neurology Unit, University of Verona, Verona, Italy

3 Department of Pathology and Diagnostic, Radiology Unit, Verona, Italy

4 Department of Surgery, Dentistry, Paediatrics and Gynaecology, Otolaryngology Unit, University of Verona, Verona, Italy

5 Department of Diagnostic and Public Health, Microbiology Section, University of Verona, Verona, Italy

6 Department of Medical Sciences, Neurology Unit, Cattinara Hospital, University of Trieste, Trieste, Italy

\section{Introduction}

First emerging in China in December 2019, the coronavirus disease 2019 (COVID-19), due to SARS-CoV-2 virus, quickly spread around the world, causing more than $200,000,000$ of cases and more than 4,000,000 deaths. In affected patients, a sudden chemosensory deficit was reported as one of the main symptoms documented retrospectively or through different tests. In particular, different studies reported chemosensory dysfunction prevalence with wide ranges, between 3 and $98 \%$ for anosmia and between 6 and $93 \%$ for taste dysfunctions [1]. Overall chemosensory impairment was reported persisting in a range around 7 to $37 \%$ of patients [2,3]. Here, we report on a deep investigation of a very young patient referred for persistent anosmia and taste disturbances, several months after the infection. The patient underwent olfactory and gustatory evaluations through validated tests, detailed neuroimaging and 
neurophysiologic assessments, and olfactory mucosa swabbing for microbiological and immunocytochemical analyses. The olfactory swabbing procedure allows a gentle collection of olfactory neuroepithelial cells, permitting a good preservation of their native cellular morphology and enabling olfactory neurons recognition. These neurons are typical bipolar cells, clearly different from other harvested cellular components [4].

\section{Case presentation}

A 17-year-old woman was referred to our chemosensory research clinic for persistent anosmia, occasional parosmia, and dysgeusia. These symptoms suddenly occurred 4 months before, at the beginning of March 2020, after a fever $\left(\sim 37.5^{\circ} \mathrm{C}\right)$ together with cough and conjunctivitis. Due to the Italian medical congested scenario for COVID19 , she could perform nasal-oropharyngeal swab for the SARS-CoV-2 molecular testing, only 1 month after clinical onset. Swab analysis resulted negative. The serological test, performed at the end of May, was positive for SARS-CoV-2 anti-nucleocapsid IgG antibodies, hence confirming the previous exposure to SARS-CoV-2. Chemosensory evaluation was then carried out through validated tests (see details on Supplementary material file).

Olfactory assessment was performed through the Sniffin' Sticks Extended Test (SSET). Results showed a composite TDI (threshold, discrimination, identification) score indicating functional anosmia (Table 1). The patient reported having had no olfactory sensation during the test, except for stinging sensations, due to the simultaneous stimulation of trigeminal fibers.

Gustatory evaluation was performed by both the whole mouth test (WMT) and the taste strips test (TST). These tests do not include umami-savory taste, since this taste quality has been found to be poorly conceptualized by the European

Table 1 Sniffin' Sticks Extended test (SSET) results for single olfactory domains and for the global score (TDI score), both for patient (17 years old) and for non-COVID-19 healthy controls, matched for age and sex (both females, 23 years old). Normosmia, TDI $\geq 30.5$; hyposmia, TDI $<30.5$; functional anosmia, TDI $\leq 16.5$

Sniffin' Sticks Extended test (SSET)

Patient Threshold Discrimi- Identification TDI score

(T) nation (I)

(D)

\begin{tabular}{rllll}
\hline 1rst evaluation & 2.25 & 5 & 5 & 12.25 \\
2nd evaluation & 2.5 & 9 & 4 & 15.5 \\
3rd evaluation & 7.5 & 15 & 11 & 33.5 \\
Control 1 & 7.5 & 13 & 16 & 36.5 \\
Control 2 & 7.25 & 13 & 14 & 34.25 \\
\hline
\end{tabular}

countries [5, 6] . Results showed hypogeusia (TST:7), with a preserved ability to identify the bitter and sour tastes, but not the sweet and salty ones. In particular, after a sweet stimulus, the evoked sensation was always bitter and none of the four administered strips was correctly identified, while after the salty stimulus, the evoked sensation was sour except for one strip in which the perceived sensation was bitter. In addition, she reported feeling of an unpleasant bitter taste during all day, often accompanied by nausea. Furthermore, she referred that most of foods elicited a bad strange taste.

Nine months after the onset, the patient underwent a second evaluation. She still complained of chemosensory disturbances; thus, further olfactory and gustatory evaluations were carried out. In particular, she complained of persistent parosmia and referred the occurrence of episodes of nausea causing difficulties in choosing food. SSET showed functional anosmia (Table 1). While at the first evaluation all the olfactory sensations were trigeminal-like (i.e., stinging, tickling, cooling) and only 5 correct identifications were recorded, at the second assessment, she referred to perceive 15 out of 16 sniffed odors but with a poor identification ability ( 4 correct identifications out of 16 total odors, often considered as unpleasant). At the second evaluation also a retronasal olfactory test was performed [7], although, after three sample administrations, the patient was not able to continue because of a sudden bout of nausea and vomit. Among the three administrations, only one was correctly identified, i.e., the coffee powder, while canella and vanilla were perceived as tingling/stinging sensations. Gustatory assessment showed normogeusia both at the WMT and TST (TST:14). Neuroimaging (3 T MRI) (Fig. S1) with special sequences for olfactory cortex, bulbs, and sulcus and neurophysiologic assessment by high-density EEG showed no anomalies (see details on Supplementary material file).

Thereafter, the patient underwent olfactory swabbing to identify abnormalities in olfactory neuroepithelium. Nasal swabbing was performed after chemosensory assessment, after written informed consent was obtained. Two nonCOVID-19 and age-matched healthy subjects were used as controls (both 23-year-olds). Controls showed normosmia and normogeusia (TDI:36.5 and 34.25; TST:14 and 15). An expert otolaryngologist explored nasal cavities using a $30^{\circ}$ rigid endoscope to detect anatomical abnormalities. Olfactory swabbing was performed at the level of the nasal vault, using a flocked swab (FLOQSwabs®; Copangroup, Brescia, Italy). An aliquot of cytological samples was tested for SARS-CoV-2 molecular analysis and the result was negative. To ensure that neuronal cells were present in our samples, immunostaining with neuron-specific class III $\beta$-tubulin marker (TUJ-1), which has been shown to label mature and immature neurons of the human olfactory epithelium, was performed $[4,8,9]$. In addition, we evaluated also the angiotensin-converting enzyme 2 (ACE2) expression using 
antibody directed to ACE2. ACE2 is commonly considered as the receptor for cellular entry adopted by the SARSCoV-2 [10]. The epithelial marker pan-cytokeratin (PCK) was used to look for the ACE2 expression in all the epithelial cells $[4,11]$. As we previously described, PCK antibody reveals a positive signal in all the olfactory epithelial cells neuronal and non-neuronal [4].

Immunocytochemistry showed the presence of olfactory neurons, either in patient or in controls (Fig. 1). ACE2 high expression was revealed in columnar-shaped cells (i.e., sustentacular cells) mainly at the apical cell membrane and at the upper cytoplasmic compartment, as previously reported [12]. Intriguingly, a less intense but sharp ACE2 dot-like cytoplasmic positivity was also detected in neuronal-shaped cells (Fig. 2). The same expression was found in patient- and age-matched controls.

Fifteen months after the onset, the patient underwent a third evaluation. Olfaction was referred to be improved but parosmia phenomena persisted; hence, some foods elicited nausea yet. SSET showed normosmia (Table 1). In particular, all the three olfactory domains improved (i.e., threshold, discrimination, identification) and identification ability increased to 11 correct identifications (Table 1). Gustatory assessment results showed normogeusia (TST: 14) with persistent dysgeusia leading to the exclusion of some foods.

\section{Discussion}

Olfactory impairment is a common symptom of upper respiratory tract post-viral infections. Interestingly, recent studies showed that olfactory validated tests could discriminate between COVID-19 and patients with acute cold, with high sensitivity and specificity, highlighting the importance of a reliable olfactory evaluation to reveal a deficit. In particular, the identification test was reported to be able to discriminate the two clinical conditions, since the overall score was significantly lower in the COVID-19 group compared to patients with acute cold [13, 14].

Here, olfaction was severely impaired (i.e., anosmia). The deficit involved all the three olfactory components (i.e., threshold, discrimination, identification), persisting at 9 months after the onset, while parosmia and dysgeusia phenomena were revealed also during the last assessment at 15 months.
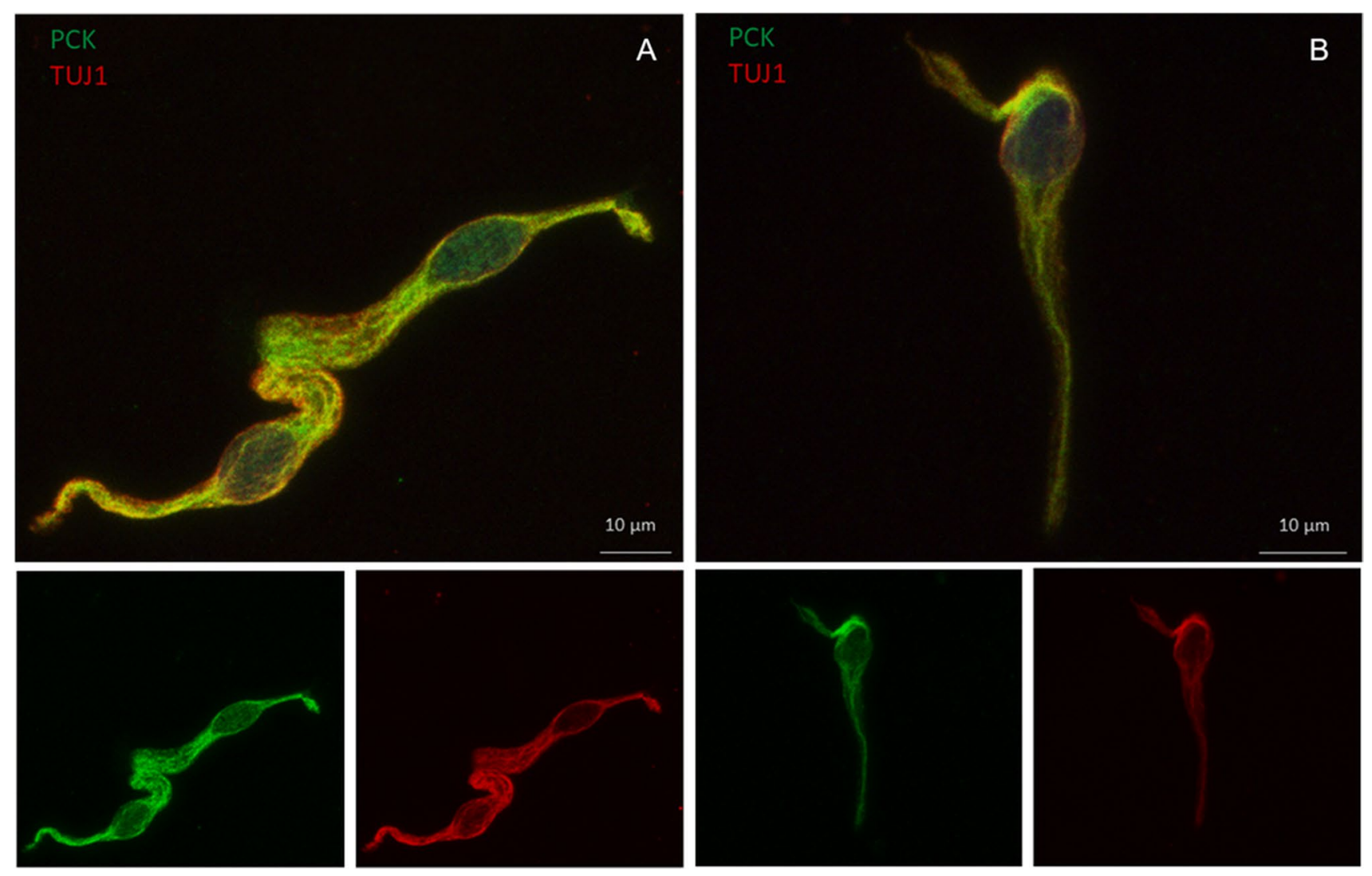

Fig. 1 Patient (A) and control (B) neuronal-shaped cells expressing the $\beta$ III tubulin (TUJ1) neuronal marker as well as the pan-cytokeratin (PCK) epithelial marker 


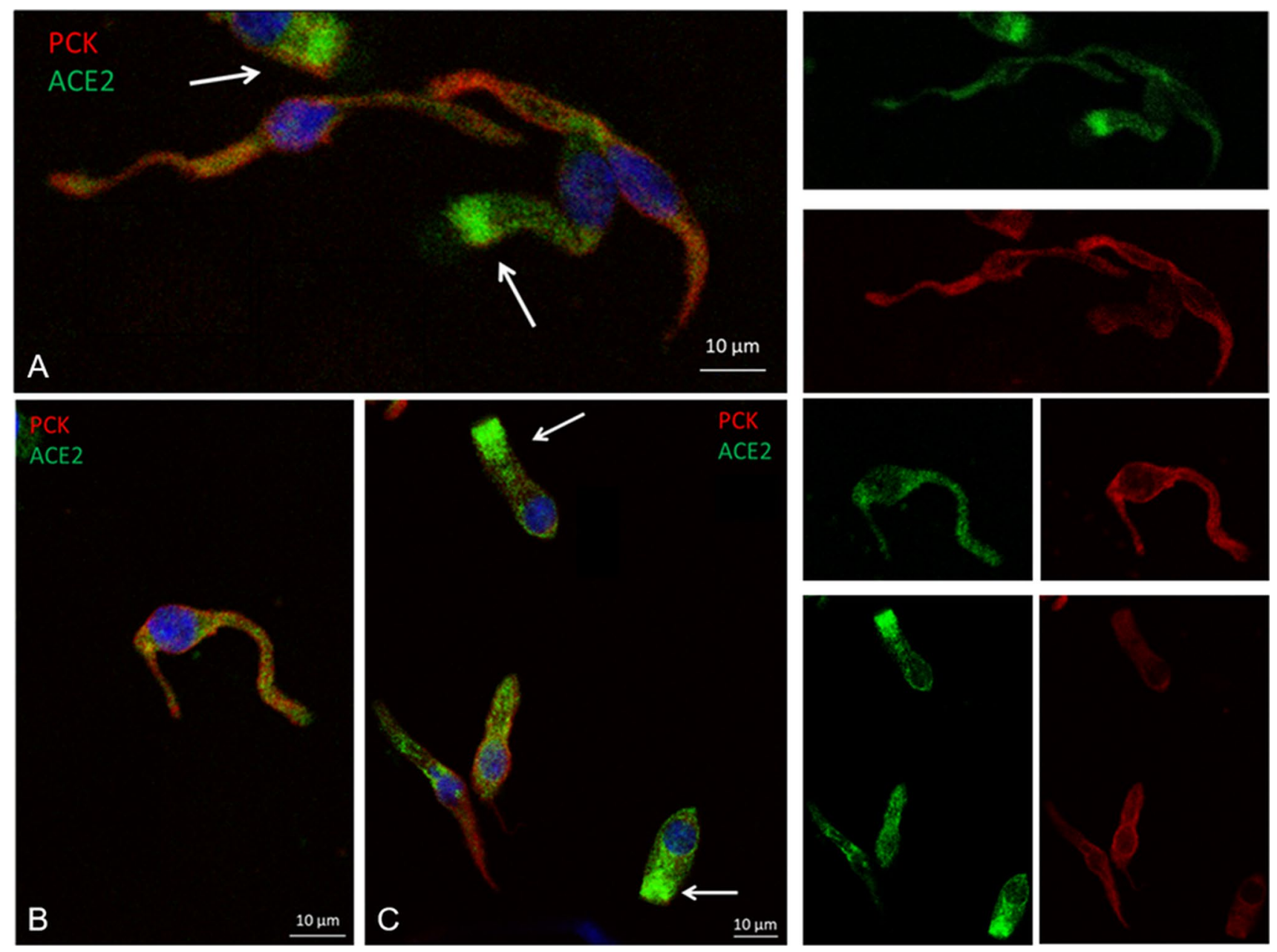

Fig. 2 Columnar-shaped cells expressing ACE2 protein mainly at the cellular apical membrane and the upper cytoplasmic compartment (arrows) and neuronal-shaped cells showing ACE2 dot-like cytoplasmic positivity. Patient (A), controls $(\mathbf{B}, \mathbf{C})$

Recent reports showed that smell recovery generally occurs within few weeks after infection, although not all patients have a full chemosensory recovery [15-17]. A persistent olfactory dysfunction was reported after a mean of 37-62 days or slightly longer after the onset [2, 18-21], and, to our knowledge, this is the first report highlighting an important smell deficit in a very young patient after COVID19 , documented trough validated tests and persisting for more than 200 days.

Notably, together with the high ACE2 expression in sustentacular cells, we observed a sharp dot-like expression in neuronal-shaped cells. This new finding might suggest that olfactory impairment could also occur through a SARS$\mathrm{CoV}-2$ direct or indirect involvement of olfactory neurons. Besides, a little expression of the ACE2 transcript was recently shown in olfactory neurons [22]. Indeed, a recent post-mortem study clearly showed SARS-CoV-2 into the human olfactory neurons [9]. This result is in line with previous experimental works showing that the SARS-CoV-1 might reach the brain via olfactory nerves [23] and cranial neuritis possibly linked to a direct viral injury of the nervous structures through olfactory fibers was recently reported [24]. Notably, ACE2 was also observed into the human olfactory bulb [25, 26].

A recent case report, investigating a 63-year-old woman with persistent anosmia for more than 3 months after SARSCoV-2 infection, showed in olfactory mucosa biopsy a significant epithelium disruption and desquamation, in the absence of central involvement [11]. These previously reported histopathological findings are in agreement with our cytological results and support the hypothesis of a possible peripheral viral damage occurring in this case.

In addition, olfactory swabbing procedure, described in details in our previous work [4], might represent a complementary and useful non-invasive approach to investigate single cell features in a so complex pseudostratified epithelium, at variance with the bioptic procedure that allows the total mucosa analysis. In addition, this technique allows to 
preserve the native cellular morphology, distinguishing the large and columnar cells from the thin and fused-shaped olfactory neurons.

Regarding taste, a gustatory deficit was documented, although a full resolution was revealed at 9 months after the onset, but with persistent dysgeusia phenomena influencing food choice.

The recent report of Huart and co-workers shows that the taste disturbances linked to SARS-CoV-2 infection might be due to a real taste impairment and not solely to a retronasal olfactory dysfunction [13]. Moreover, a recent case series study reported a mild facial nerve defect in the right lower branch with asymmetry of the latency and amplitude conduction and in one case this deficit was accompanied by sweet and salty taste ageusia on the right side of the tongue [27].

Interestingly, according to the findings of these works, we found that in particular sucrose was never detected to any concentration and the perception was referred as bitter. Sweet is a robust archaic taste linked to energy intake as well as bitter is for toxic detection. Rarely this perception is so clearly impaired also at supra-threshold level. Sweet and bitter receptors are G protein-coupled receptors (GPCRs), belonging to the same class of those expressed in the lung, supposed to be a possible virus entry [28]. The same mechanism could occur even in the oral cavity [13]. In addition, given that a recent transcriptomic analysis shows ACE2 and transmembrane serine proteases (TMPRSS2) expressed in salivary glands, also saliva could play a role in the gustatory deficit [29]. Regarding the salty taste referred as sour, this wrong perception might be partly due to the similarity of the two taste qualities, producing both a slight tingling/stinging sensation on the tongue, as previously reported [30].

No imaging and neurophysiologic anomalies were associated with the chemosensory deficit, suggesting a peripheral involvement. Nevertheless, as MRI was performed 4 months after onset, we cannot exclude that it would have revealed abnormalities if performed in the acute phase. In addition, a recent functional MRI study showed orbitofrontal cortex involvement in a patient with persistent cacosmia and cacogeusia after COVID-19 infection, in spite of normal brain MRI [31].

\section{Conclusion}

Summarizing, it emerges that in a very young COVID-19 case with a mild clinical picture and no comorbidities, a deep chemosensory deficit, not fully recovered at 15 months after the onset, with persisting parosmia and dysgeusia phenomena, caused a relevant impact on the daily life. Olfaction resulted to be mainly impaired, and ACE2 detection in both sustentacular and neuronal-shaped cells could explain possible several virus damage peripheral mechanisms. The quantitative gustatory dysfunction even if less persisting, for the aforementioned features, could be related to a primary gustatory system involvement.

Multicentric studies, assessing chemosensory function for an extended period of time on a consistent number of subjects, are necessary to expand the knowledge in this field and unravel all the different clinical pictures, especially in young subjects. Validated smell and taste tests are always highly recommended to document and characterize the deficit also in a longitudinal fashion.

Supplementary Information The online version contains supplementary material available at https://doi.org/10.1007/s10072-021-05635-y.

Acknowledgements The authors thank Dr. Santina Castriciano (Copangroup) for donating olfactory flocked swabs.

Funding This study was partially supported by the Cariverona Foundation, ENACT project VIRO-COVID (Prof. Davide Gibellini).

\section{Declarations}

Ethics approval All the procedures were undertaken with the understanding and written consent of each subject, and the study conforms with the World Medical Association Declaration of Helsinki. The olfactory swabbing was performed following the approval of the Ethical Committee of University Hospital of Verona (Prot.n.28917 June, 15th, 2012).

Consent for publication Written informed consent was collected from both patient and controls for the inclusion of anonymous clinical data in a scientific publication, in accordance with the Declaration of Helsinki.

Conflict of interest The authors declare no competing interests.

\section{References}

1. von Bartheld CS, Hagen MM, Butowt R (2020) Prevalence of chemosensory dysfunction in COVID-19 patients: a systematic review and meta-analysis reveals significant ethnic differences. ACS Chem Neurosci 11(19):2944-2961

2. Chiesa-Estomba CM, Lechien JR, Radulesco T, Michel J, Sowerby LJ, Hopkins C, Saussez S (2020) Patterns of smell recovery in 751 patients affected by the COVID-19 outbreak. Eur J Neurol 27:2318-2321

3. Moein ST, Hashemian SM, Tabarsi P, Doty RL (2020) Prevalence and reversibility of smell dysfunction measured psychophysically in a cohort of COVID-19 patients. Int Forum Allergy Rhinol 10(10):1127-1135

4. Brozzetti L, Sacchetto L, Cecchini MP, Avesani A, Perra D, Bongianni M, Portioli C, Scupoli M, Ghetti B, Monaco S, Buffelli M, Zanusso GL (2020) Neurodegeneration-associated proteins in human olfactory neurons collected by nasal brushing. Front Neurosci mar 5;14:145.eCollection 2020

5. Landis BN, Welge-Luessen A, Brämerson A et al (2009) "Taste strips" - a rapid, lateralized, gustatory bedside identification test based on impregnated filter papers. J Neurol 256:242-248 
6. Cecchini MP, Knaapila A, Hoffmann E et al (2019) A cross-cultural survey of umami familiarity in European countries. Food, Qual \& Pref 74:172-178

7. Heilmann S, Strehle G, Rosenheim K, Damm M, Hummel T (2002) Clinical assessment of retronasal olfactory function. Arch Otolaryngol Head Neck Surg 128:414-418

8. Holbrook EH, Wu E, Curry WT, Lin DT, Schwob JE (2011) Immunohistochemical charachterization of human olfactory tissue. Laryngoscope 121:1687-1701

9. Meinhardt J, Radke J, Dittmayer C, Franz J, Thomas C, Mothes R, Laue M, Schneider J, Brünink S, Greuel S, Lehmann M, Hassan O, Aschman T, Schumann E, Chua RL, Conrad C, Eils R, Stenzel W, Windgassen M et al (2020) Olfactory transmucosal SARS-CoV-2 invasion as a port of central nervous system entry in individuals with COVID-19. Nat Neurosci 24:168-175

10. Hoffmann M, Kleine-Weber H, Schroeder S, Krüger N, Herrler T, Erichsen S, Schiergens TS, Herrler G, Wu NH, Nitsche A, Müller MA, Drosten C, Pöhlmann S (2020) SARS-CoV-2 cell entry depends on ACE2 and TMPRSS2 and is blocked by a clinically proven protease inhibitor. Cell 181:271-280.e8

11. Vaira LA, Hopkins C, Sandison A, Manca A, Machouchas N, Turilli D, Lechien JR, Barillari MR, Salzano G, Cossu A, Saussez S, De Riu G (2020) Olfactory epithelium histopathological findings in long-term COVID-19 related anosmia. J Laryngol Otol 134:1123-1127

12. Brann DH, Tsukahara T, Weinreb C, Lipovsek M, Van den Berge K, Gong B, Chance R, Macaulay IC, Chou HY, Fletcher RB, Das D, Street K, Roux de Bezieux H, Choi YG, Risso D, Dudoit S, Purdom E, Mill J, Abi Hachem R, Matsunami H, Logan DW, Goldstein BJ, Grubb MS, Ngai J, Datta SR (2020) Non-neuronal expression of SARS-CoV-2 entry genes in the olfactory system suggests mechanisms underlying COVID-19-associated anosmia. Sci Adv 6(31):eabc5801

13. Huart C, Philippot C, Konstantinidis A, Altundag A, Whitcroft KL, Trecca EMC, Cassano M, Rombaux P, Hummel T (2020) Comparison of COVID-19 and common cold chemosensory dysfunction. Rhinology 58:623-625

14. Haehner A, Draf J, Dräger S, de With K, Hummel T (2020) Predictive value of sudden olfactory loss in the diagnosis of COVID19. ORL J Otorhinolaryngol Relat Spec 82:175-180

15. Konstantinidis I, Delides A, Tsakiropoulou E, Maragoudakis P, Sapounas S, Tsiodras S (2020) Short-term follow-up of selfisolated COVID-19 patients with smell and taste dysfunction in Greece: two phenotypes of recovery ORL J Otorhinolaryngol Relat spec 82:295-303

16. Iannuzzi L, Salzo AE, Angarano G, Palmieri VO, Portincasa P, Saracino A, Gelardi M, Dibattista M, Quaranta N (2020) Gaining back what is lost: recovering the sense of smell in mild to moderate patients after COVID-19. Chem Sens 45:875-881

17. Otte MS, Eckel HNC, Poluschkin L, Klussmann JP, Luers JC (2020) Olfactory dysfunction in patients after recovering from COVID-19. Acta Otolaryngol 140:1032-1035

18. Li J, Long X, Zhu C, Wang H, Wang T, Lin Z, Li J, Xiong N (2020) Olfactory dysfunction in recovered COVID-19 patients. Mov Disord 35:1100-1101

19. Le Bon SD, Pisarski N, Verbeke J, Prunier L, Cavelier G, Thill MP, Rodriguez A, Dequanter D, Lechien JR, Le Bon O, Hummel T, Horoi M (2021) Psychophysical evaluation of chemosensory functions 5 weeks after olfactory loss due to COVID-19: a prospective cohort study on 72 patients. Eur Arch Otorhinolaryngol 278:101-108

20. Lechien JR, Journe F, Hans S, Chiesa-Estomba CM, Mustin V, Beckers E, Vaira LA, De Riu G, Hopkins C, Saussez S (2020) Severity of anosmia as an early symptom of COVID-19 infection may predict lasting loss of smell. Front Med 7:582802

21. Lechien JR, Chiesa-Estomba CM, Beckers E, Mustin V, Ducarme M, Journe F, Marchant A, Jouffe L, Barillari MR, Cammaroto G, Circiu MP, Hans S, Saussez S (2021) Prevalence and 6-month recovery of olfactory dysfunction: a multicenter study of 1363 COVID-19 patients. J Int Med (Jan 5). https://doi.org/10.1111/ joim. 13209

22. Fodoulian L, Tuberosa J, Rossier D, Boillat M, Kan C, Pauli V, Egervari K, Lobrinus JA, Landis BN, Carleton A, Rodriguez I (2020) SARS-CoV-2 receptors and entry genes are expressed in the human olfactory neuroepithelium and brain. iScience 23:101839

23. Nedland J, Meyerholz DK, Moore S, Cassell M, Perlman S (2008) Severe acute respiratory coronavirus infection causes neuronal death in the absence of encephalitis in mice transgenic for human ACE2. J Virol 82:7264-7275

24. De Gennaro R, Gastaldo E, Tamborino C, Baraldo M, Casula N, Pedrali M, Iovino S, Michieletto L, Violo T, Ganzerla B, Martinello I, Quatrale R (2021) Selective cranial multineuritis in severe COVID-19 pneumonia: two cases and literature review. Neurol Sci 42(5):1643-1648

25. Chen R, Wang K, Yu J, Howard D, French L, Chen Z, Wen C, Xu Z (2021) The spatial and cell-type distribution of SARS-CoV-2 receptor ACE2 in the human and mouse brains. Front Neurol Jan20;11:573095.eCollection 2020

26. Klingenstein $M$, Klingenstein $S$, Neckel PH, Mack AF, Wagner AP, Kleger A, Liebau S, Milazzo A (2021) Evidence of SARS$\mathrm{CoV} 2$ entry protein ACE2 in the human nose and olfactory bulb. Cells Tissues Organs 22:1-10

27. Manganotti P, Bellavita G, D'Acunto L, Tommasini V, Fabris M, Sartori A, Bonzi L, Stella AB, Pesavento V (2021) Clinical and cerebrospinal liquor analysis to detect Guillan-Barrè syndrome and polyneuritis cranialis in COVID-19 patients: a case series. $\mathrm{J}$ Med Virol 93:766-774

28. Singh Y, Gupta G, Satjia S, Pabreja K, Chellappan DK, Dua K (2020) COVID-19 transmission through host cell directed network of GPCR. Drug Dev Res 81:647-649

29. Song J, Li Y, Huang X, Chen Z, Li Y, Liu C, Chen Z, Duan X (2020) Systematic analysis of ACE2 and TMPRSS2 expression in salivary glands reveals underlying transmission mechanism caused by SARS-CoV-2. J Med Virol 92:2556-2566

30. Mueller C, Kallert S, Renner B (2003) Quantitative assessment of gustatory function in a clinical context using impregnated "taste strips". Rhinology. 41:2-6

31. Ismail II, Gad KA (2021) Absent blood oxygen level-dependent functional magnetic resonance imaging activation of the orbitofrontal cortex in a patient with persistent cacosmia and cacogeusia after COVID-19 infection. JAMA Neurol 78(5):609-610

Publisher's note Springer Nature remains neutral with regard to jurisdictional claims in published maps and institutional affiliations. 